Objectives: To comprehensively investigate the intestinal mucosa cytokine production and $\mathrm{DC}, \mathrm{T}$ and $\mathrm{B}$ cell responses to human gut microbiota associated with new-onset RA.

Methods: We utilized in vitro cultures of mucosal-like DCs (differentiated from bone marrow cells) and primary splenic DCs, as well as ex vivo cultures of healthy human intestinal biopsies, cultured in the presence of heat-killed fecal microbiota from either NORA or control donors. Furthermore, we performed studies in humanized mice carrying intestinal NORA microbiota, to study the effect on immune response during homeostasis and upon joint inflammation during collagen-induced arthritis (CIA).

Results: In 24h DC cultures, NORA fecal microbiota more potently induced the expression of co-stimulatory molecules CD40 and CD80, and this enhanced DC maturation was partially mediated through TLR4 as demonstrated using the TLR4 antagonist TAK242. Interestingly, HC and NORA fecal microbiota differentially induced IL-12 and IL-6 production, with significantly enhanced IL-6 and reduced IL-12 secretion by the NORA microbiome. Furthermore, in ex vivo cultures of human ileum biopsies, the production of IL-1 and IL-33, as well as IL-23/Th17 cytokines IL-23, IL-22, and GM-CSF, were significantly increased by NORA-derived microbiome. Interestingly, in the small intestine lamina propria (SILP) of NORA-colonized mice, we observed enhanced Th17 polarization, increased innate GM-CSF expression and higher B cell CD40 and IgA levels during homeostasis. To study whether colonization with $\mathrm{HC}$ and NORA microbiota alters arthritis development, humanized mice and controls (mock, autologous, $\mathrm{HC}$ and NORA microbiota) were used in a CIA experiment. Macroscopic scoring of the arthritis severity at weekly intervals demonstrated that arthritis severity was significantly enhanced in NORA-colonized mice compared to HC-colonization and mock controls.

Conclusion: Our data reveal that NORA microbiota, in addition to the previously described Th17 differentiation, induce higher levels of GM-CSF and B cell $\lg A$ in LP and have increased potential to aggravate arthritis through the activation of TLR4.

References:

[1] Scher et al., eLife 2013; Maeda Y et al., Arthritis \& rheumatology 2016; Zhang

X et al., Nature medicine 2015; Chen J et al., Genome Med 2016

Disclosure of Interests: Marije Koenders: None declared, Heather EvansMarin: None declared, Joyce Aarts: None declared, Parvathy Girija: None declared, Rebecca Rogier: None declared, Sergei Koralov: None declared, Julia Manasson: None declared, Peter van der Kraan: None declared, Shahla Abdollahi-Roodsaz: None declared, Jose Scher Consultant of: Novartis, Janssen, UCB, Sanofi.

DOI: 10.1136/annrheumdis-2020-eular.5269

\section{THU0080 PRECLINICAL CHARACTERIZATION OF TLL018, A NOVEL, HIGHLY POTENT AND SELECTIVE JAK1/TYK2 INHIBITOR FOR TREATING AUTOIMMUNE DISEASES}

X. Liu ${ }^{1}$, F. Tan ${ }^{1}$, C. Liang ${ }^{1} .{ }^{1} T L L$ Pharmaceutical LLC, Iselin, United States of America

Background: Janus kinases (JAKs) are important regulators of intracellular responses triggered by many key proinflammatory cytokines and are clinically validated therapeutic targets for treating various autoimmune diseases. However, current approved JAK inhibitors failed to achieve maximal clinical benefit in part due to their unfavorable selectivity for individual JAKs such as JAK2 and/ or JAK3, leading to dose-limiting toxicities or severe toxicities (e.g., thrombosis, anemia, immune suppression). Selective inhibition of JAK1 and/or TYK2 may minimize or avoid some of the toxicities and potentially offer a better therapeutic window for treating autoimmune diseases. No highly selective JAK1/TYK2 inhibitor has been reported to date.

Objectives: Discovery of a highly selective JAK1/TYK2 inhibitor that maximally avoids JAK2 and JAK3 inhibition. We described preclinical characterization of a novel, highly potent and selective JAK1/TYK2 inhibitor TLL018 and its potential utility in treating autoimmune diseases such as rheumatoid arthritis (RA).

Methods: Using predicting SAR, TLL018 was designed to achieve exquisite selectivity for both JAK1 and TYK2 while sparing JAK2, JAK3 and other human kinases. Its enzyme and cell activities, kinase selectivity, and in vivo efficacy were assessed in a battery of relevant enzyme, cell and whole blood assays, and in vivo arthritis animal models. Additional preclinical DMPK and toxicology studies were conducted to support its clinical development.

Results: TLL018 is a highly potent and selective, orally bioavailable JAK1/TYK2 inhibitor against JAK1 $\left(\mathrm{IC}_{50}=4 \mathrm{nM}\right)$ and TYK2 $\left(\mathrm{IC}_{50}=5 \mathrm{nM}\right)$ as measured in in vitro kinase assays with ATP concentrations at individual $\mathrm{Km}$. Its potency against JAK2 or JAK3 is greater than $1 \mu \mathrm{M}$. Profiling against a panel of over 350 human kinase showed that TLL018 is exclusively selective for JAK1 and TYK2, with $\geq$ 90-fold selectivity against all other kinases tested. TLL018 exhibited potent cellular activity for JAK1-mediated IL-6 signaling $\left(\mathrm{IC}_{50}=0.6 \mu \mathrm{M}\right)$ with greater than 100 fold selectivity against JAK2-mediated cytokine (e.g., TPO) signaling in human whole blood-based assays.

Oral administration of TLL018 demonstrated dose-dependent efficacy in commonly studied rat adjuvant-induced arthritis ( $\mathrm{rAIA}$ ) model and mouse collagen-induced arthritis ( $\mathrm{mClA}$ ) model. Significant inhibition of inflammation, bone resorption, splenomegaly and body weight change was observed in adjuvant-induced disease in rats. In addition, significant inhibition of inflammation, cartilage destruction, bone resorption and histological signs was demonstrated in collagen-induced arthritis in mice. Noticeably, TLL018 exhibited significant anti-inflammation activity at doses that only blocked JAK1 and TYK2 and exerted little inhibition of JAK2 and JAK3.

In support of clinical development of TLL018, preclinical ADME and PK studies and IND-enabling toxicology and safety pharmacology studies were completed confirming that TLL018 possesses excellent ADME and PK properties, and exhibits a clean on-target safety profile.

Conclusion: TLL018 is a highly potent and selective JAK1/TYK2 inhibitor that demonstrated excellent efficacy and tolerability in relevant mouse and rat arthritis models. The collective data of its preclinical pharmacology, PK and toxicology showed a favorable pharmaceutical profile, further supporting its development for treating autoimmune diseases including RA. Clinical evaluation of TLL018 is ongoing.

Disclosure of Interests: Xiangdong Liu Shareholder of: I own shares of TLL Pharmaceutical LLC, Employee of: I am employed by TLL Pharmaceutical LLC, Fenlai Tan Shareholder of: I own shares of TLL Pharmaceutical LLC, Employee of: I am employed by TLL Pharmaceutical LLC, Chris Liang Shareholder of: I own shares of TLL Pharmaceutical LLC, Employee of: I am employed by TLL Pharmaceutical LLC DOI: 10.1136/annrheumdis-2020-eular.1547

\section{THU0081 MIR-17-5P REDUCES INFLAMMATION AND BONE EROSIONS IN COLLAGEN INDUCED ARTHRITIS MICE AND DIRECTLY TARGETS THE JAK-STAT PATHWAY IN RHEUMATOID ARTHRITIS FIBROBLAST-LIKE SYNOVIOCYTES.}

A. Najm ${ }^{1,2}$, F. M. Masson ${ }^{1}$, P. Preuss ${ }^{1,2}$, S. Georges ${ }^{1}$, B. Ory ${ }^{1}$, T. Quillard $^{1}$, D. Veale ${ }^{3}$, U. Fearon ${ }^{4}$, B. Le Goff ${ }^{1,2}$, F. Blanchard ${ }^{1}$. ${ }^{1}$ Nantes Faculty of Medicine, PHY-OS Laboratory INSERM UMR 1238, Nantes, France; ${ }^{2}$ Nantes University Hospital, Department of Rheumatology, Nantes, France; ${ }^{3}$ The Centre for Arthritis and Rheumatic Diseases, St. Vincent's University Hospital, Department of Rheumatology, Dublin, Ireland; ${ }^{4}$ Trinity Biomedical Sciences Institute, Trinity College Dublin., Molecular Rheumatology, Dublin, Ireland

Background: micro-RNAs (miR) are strong regulators of gene expression. Their involvement in RA key cytokines pathway regulation entitles them as important players in RA pathophysiology. The miR 17-92 cluster has been widely studied in cancer as they regulate cell apoptosis.

Objectives: The aims of this study were to screen miR 17-92 cluster's expression in different RA phenotypes (erosive and non erosive), further elucidate the mechanisms and direct targets involved in miR-17-5p anti-inflammatory role and to investigate miR-17-5p therapeutic effect in arthritis.

Methods: A miR array was performed in synovial tissue from naïve erosive and non-erosive RA patients. Intra-articular delivery of miR-17 lipoplex was performed in collagen induced arthritis model in mice. Clinical, histological and structural effects were studied over the course of arthritis. In depth studies of miR-17 mechanisms of action were performed in primary RA-FLS isolated from RA synovial tissue.

Results: Among others, miR-17-5p expression was reduced in erosive RA miR-17 transfection in arthritic paws significantly reduced clinical inflammation Moreover, synovial B cells, T cells, macrophages and polynuclear neutrophils infiltrates were significantly reduced. Structural damage was also decreased as shown by a reduction in the number of osteoclasts and erosion score by CT analysis. Pro-inflammatory cytokines of the IL- 6 family, STAT3 target genes and IL-1 $\beta$ expression were also significantly reduced, but not TNF-alpha. miR17 directly targeted the 3'-untranslated region of STAT3 and JAK1. STAT3 and JAK1 mRNA and protein expression were reduced in RA-FLS following miR-17 transfection. STAT3 and JAK1 mRNA and activation of STAT3 as assessed by immunohistochemistry were also reduced in injected paws.

Conclusion: We demonstrate an anti-inflammatory and anti-erosive role of miR-17 in vivo. This effect involves the suppression of the IL-6 family autocrine amplifying loop through the direct targeting of JAK1 and STAT3 as shown in RA-FLS. 